\title{
A bi-objective model for emergency services location-allocation problem with maximum distance constraint
}

\author{
Mansoureh Haj Mohammad Hosseini ${ }^{{ }^{*}}$, Mohammad Saeed Jabal Ameli ${ }^{\mathbf{a}}$
}

${ }^{a}$ Department of Industrial Engineering, Iran University of Science \& Technology, Tehran, Iran

\begin{tabular}{|c|c|}
\hline A R T I C L E I N F O & A B S T R A C T \\
\hline $\begin{array}{l}\text { Article history: } \\
\text { Received } 26 \text { August } 2010 \\
\text { Received in revised form } \\
12 \text { December } 2010 \\
\text { Accepted } 15 \text { December } 2010 \\
\text { Available online } \\
15 \text { December } 2010\end{array}$ & $\begin{array}{l}\text { In this paper, a bi-objective mathematical model for emergency services location-allocation } \\
\text { problem on a tree network considering maximum distance constraint is presented. The first } \\
\text { objective function called centdian is a weighted mean of a minisum and a minimax criterion } \\
\text { and the second one is a maximal covering criterion. For the solution of the bi-objective } \\
\text { optimization problem, the problem is split in two sub problems: the selection of the best set of } \\
\text { locations, and a demand assignment problem to evaluate each selection of locations. We }\end{array}$ \\
\hline $\begin{array}{l}\text { Keywords: } \\
\text { Centdian } \\
\text { Maximal covering }\end{array}$ & $\begin{array}{l}\text { propose a heuristic algorithm to characterize the efficient location point set on the network. } \\
\text { Finally, some numerical examples are presented to illustrate the effectiveness of the proposed } \\
\text { algorithm. }\end{array}$ \\
\hline
\end{tabular}

\section{Introduction}

One of the most important issues judging the performance of emergency services location problem is equity. For considering equity, authors usually consider the objective of minimizing the maximum distance or response time and use the P-center criterion for formulating such situations. In the Pcenter problem, the objective is locating $p$ facilities on a network of demands so that the maximum distance of all users from their corresponding facilities is minimized. A further significant factor in locating emergency services is spatial efficiency. We use the P-median criterion to address the spatial efficiency. The P-median problem consists of locating $p$ facilities in a given network which satisfy demand points in such a way that the total sum of weighted distances between each demand point and its corresponding facility is minimized. The p-median problem emphasizes on averaging which is not considered sufficient in the context of emergency services since it often makes solutions where remote and low population density areas are discriminated against centrally situated and high

* Corresponding author. Tel: +9821-77240482

E-mail addresses: mhosseini@ind.iust.ac.ir (M. Haj Mohammad Hosseini) 
population density areas. On the other hand, the location of a facility at the center may cause a large increase in the total distance, which means no spatial efficiency (Colebrook \& Sicilia 2007). Halpern (1976) is believed to be the first who introduced the concept of the centdian. The criterion minimizes the convex combination of the center and median objective functions. This function allows exploiting jointly the main advantages of each previous problem. In this paper, we use the centdian criterion as the first objective function. To consider the impact of the emergency services we propose the second criterion. The second criterion is a measure for the distances between the demand nodes and their corresponding allocated emergency services. If an emergency service is too far from the demand node, it might be unable to provide service to the demand as it takes too much time to get there. So we consider the maximal covering (MCLP) criterion as the second objective. The maximal covering criterion consists in minimizing the penalty of missing some demands that are not able to reach an emergency service within a predefined reasonable distance.

The rest of the paper is organized as follows. In section 2, we review the literature related to emergency services location problems. In section 3, we introduce the notation, definitions and the proposed model formulated as a bi-objective optimization model. In section 4, we propose a heuristic algorithm to solve the bi-objective optimization problem. Section 5 presents numerical experiments that illustrate our methodology. Section 6 summarizes the conclusion and contribution of this work.

\section{Literature review}

The review on emergency services location can be separated into three sections depending on the objective function of the models, and this leads to covering, P-median, and P-center models.

In network location theory, the p-median and the p-center problems often are two main criteria to choose optimally of a set of $p$ points on a network. Hakimi (1964) introduced and solved these two problems. The P-median and P-center models are two widespread location models for formulating emergency facility location problems (Jia et al., 2007).

\section{1 p-median models for emergency services}

The p-median model has been applied in many emergency facility location problems. Carbone (1974) implemented a deterministic P-median model with the objective of minimizing the total traveled distance among users and fixed public facilities such as medical or day care centers. Calvo and Marks (1973) built a P-median model to locate multi-level healthcare facilities including central hospitals, community hospitals and local reception centers. Tien et al. (1983) and Mirchandani (1987) used the hierarchical P-median model for emergency service location. Paluzzi (2004) considered a p-median location model for placing emergency service facilities for the city of Carbondale in United States of America. Research on emergency service P-median based models has also been extended to incorporate the stochastic and probabilistic situations. Mirchandani (1980) developed a P-median problem to locate fire-fighting emergency units with the consideration of stochastic travel characteristics and demand patterns. Serra and Marianov (1999) considered the problem of locating a fire station for emergency services in Barcelona when there are uncertainties in demand, travel time or distance. P-median models have also been extended to solve emergency service location problems in a queuing theory context (e.g. Berman et al., 1985).

\subsection{P-center models for emergency services}

There are several works dedicated to the context of locating facilities such as emergency services centers, hospitals, fire stations, and other public facilities. Garfinkel et al. (1977) proposed a modeled to solve the $p$-center problem in order to locate a given number of emergency facilities along a road network. They also examined the basic properties of the P-center problem in their work. ReVelle and Hogan (1989) developed a P-center problem for locating the emergency facilities to minimize the maximum distance where the emergency service facility is available with the probability of $\alpha$. Research on emergency service P-center problems has also been extended to incorporate the stochastic and probabilistic situations. Hochbaum and Pathria (1998) considered the emergency 
facility location problem and with a simple assumption that the cost and the distance among locations vary for each discrete period and the objective function was to minimize the maximum distance on the network across all periods.

Talwar (2002) extended a P-center model for locating and dispatching three emergency rescue helicopters to serve the growing emergency service demands due to accidents occurring during adventure holidays.

\subsection{Covering models for emergency services}

The second objective function considered in this paper is the maximal covering criterion. Location covering problems are the most popular location models for formulating emergency facility location problems (Jia et al., 2007). The first of these models, the location set covering problem (Toregas et al., 1971) aims to determine the least number and the positions of servers on a network so that each point of demand contains, at least, one server stationed within $S$ distance units. The mentioned models do not consider the system congestion and unavailability of the facilities and there are other techniques and models, which incorporated the congestion effect by providing redundant or backup coverers (Daskin \& Stern, 1981; Bianchi \& Church, 1988). The primary objective of these models is to make sure that a backup facility is available to serve a demand area when the first facility is unavailable. There are other types of emergency service covering models which incorporate the uncertain situations. Probabilistic location techniques assume that any given ambulance may be busy once needed (Goldberg \& Paz, 1991; and Beraldi \& Ruszczynski, 2002). Daskin (2008) considered server independence and system-wide server busy probability for maximum expected coverage location problem (MEXCLP). A poplar approach to formulating stochastic emergency services covering problems is to use scenario planning. In this approach, we represent possible values for parameters that may vary over the planning horizon in different emergencies. For example, Schilling (1982) extended the MCLP by incorporating scenarios to maximize the covered demands over all possible scenarios. There are other works incorporating the idea of scenarios (Jia et al., 2007).

\subsection{The need for multi-objective model}

As we mentioned earlier, the P-center criterion may cause a large increase in the total distance, which means no spatial efficiency. Therefore, we look for the P-centdian criterion as the first objective function as an additional criterion for the proposed model of this paper. This criterion considers both equity and efficiency in one objective. Although the P-center criterion seeks to minimize the maximum distance, it does not consider the demands of the nodes, which receive service from facilities. Therefore, we consider another objective, which aims to maximize the demand that can receive service from a facility in a predefined maximum distance.

\section{Problem definition}

In this section, we describe the details of the proposed bi-objective optimization model of this paper. The proposed bi-objective model for locating emergency services incorporates the following two objectives:

(1) The first objective, the centdian objective, is the convex combination of the center, minimax, facility location and the median, minisum, facility location.

(2) The maximal covering objective, which minimizes the penalty function considered for the customers who are unable to reach a facility within a predefined maximum distance.

\subsection{Variables, indexes, and notations}

We use the following notation

$T \quad:$ an undirected tree

$V \quad$ : set of nodes 
E $\quad$ : set of edges

$n \quad$ : index for demand nodes

$m \quad$ : index for alternative sites for emergency stations

$w_{j} \quad:$ weight (demand) associated with node $\mathrm{j}, \forall j \in J$ for median objective

$u_{j} \quad$ : weight associated with node $\mathrm{j}, \forall j \in J$ for center objective

$d_{\max }: \quad$ : specifies the MCLP distance threshold

Decision variables

$u_{i j}= \begin{cases}1 & \text { if the demand of node } \mathrm{j} \text { is served by a facility in candidate location } \mathrm{i} \\ 0 & \text { otherwise }\end{cases}$

$y_{j}= \begin{cases}1 & \text { if demand node } \mathrm{j} \text { has a facility within a distance of less or equal } \mathrm{d}_{\max } \\ 0 & \text { otherwise }\end{cases}$

\section{Constants}

$d_{i j} \quad:$ the distance between nodes $i$ and $j$ is $\operatorname{denoted}(i \in I, j \in J)$

$a_{i j}= \begin{cases}1 & \text { if } \mathrm{d}_{\mathrm{ij}} \leq \mathrm{d}_{\max } \\ 0 & \text { otherwise }\end{cases}$

$z_{i} \quad$ : number of capacity units at location $i(i \in I)$

\subsection{Mathematical programming formulation of the proposed model}

With this notation, we obtain the following bi-objective nonlinear integer programming:

$$
\begin{aligned}
& \min \left(f_{1}, f_{2}\right) \\
& f_{1}=\lambda\left[\max _{j \in J} \sum_{i \in I} d_{i j} u_{i j}\right]+(1-\lambda) \cdot \sum_{j \in J} w_{j} \sum_{i \in I} d_{i j} u_{i j} \\
& f_{2}=\sum_{j \in J} w_{j}\left(1-y_{j}\right) \\
& \sum_{i \in I} u_{i j}=1, \forall j \in J \\
& x_{i}-u_{i j} \geq 0 \quad, \forall i \in I, j \in J \\
& \sum_{i \in I} a_{i j} \cdot x_{i} \geq y_{j}, \forall j \in J \\
& \sum_{j \in J} w_{j} u_{i j} \leq z_{i}, \forall i \in I \\
& u_{i j} \in\{0,1\}, \forall i \in I, \quad j \in J \\
& x_{i} \in\{0,1\}, \forall i \in I, y_{j} \in\{0,1\}, \forall j \in J
\end{aligned}
$$

Eq. (2) determines the first objective function called centdian criterion as a weighted average of minisum objective function and minimax objective function. $\lambda$ is a parameter for the centdian objective with $0<\lambda<1$. Eq. (3) determines the second objective function, which aims to minimize the total uncovered demand. Eq. (4) requires that each demand node has a assigned facility. Eq. (5) determines that only a candidate-established location can provide service, Eq. (6) ensures that demand node $j$ can be served within distance $d_{\max }$ if there is a node I within distance $d_{\max }$ with an established facility. Eq. (7) is the capacity constraint and it ensures that the demand occurring at 
location $i$ is covered by the capacity units. Finally, Eqs. (8)-(9) restrict the variables to have only binary values.

\section{Proposed solution approach}

The proposed model of this paper is formulated as a multi-objective mathematical problem (MOMP) and the solution procedure is based on the techniques used in this context where we get some Pareto optimal solutions and the collection of all the optimal solutions is called the Pareto set. The proposed MOMP of this paper uses generation technique which is the e-constraint method. The method provides a representative subset of the Pareto set which is adequate in most cases. The following is a brief explanation of e-constraint method.

\subsection{The e-constraint method}

Consider the following multi-objective problem,

$\min \left(f_{1}(x), f_{2}(x), \ldots, f_{p}(x)\right)$

subject to

$x \in S$,

where $x$ is the vector of decision variables, $f_{1}(x), f_{2}(x), \ldots, f_{p}(x)$ are the objective functions, and $\mathrm{S}$ is the feasible region. In e-constraint method, we optimize one objective function and consider other objectives as constraints. Therefore, we can write the model in following form,

$\min \left(f_{1}(x)\right)$

subject to

$f_{i+1}(x) \geq e_{i}, \quad i=1, \cdots p$

$x \in S$

Once we assign different values for $e_{i}$ we can obtain various efficient solutions. For the proposed model of this paper since we deal with only two variables, the proposed model minimizes the first objective, $f_{1}(x)$, and the objective function, $f_{2}(x)$, is added in addition to other constraints in a form of $f_{2}(x) \geq e_{1}$. In the next sections we will describe the solution procedure of the proposed model. The general strategy we apply is as follows. Given a non-negative real value $r$, the $p$-centdian is a $r$ restricted $p$-median problem on the tree $T$ that is the minimum of the sum of the weighted distances of the nodes to a $p$-median set, given that the weighted distance of each node to the $p$-median set is at most $r$. Therefore, we have,

$m(r)=\min \left\{f_{1}^{(2)} \mid w_{i} d\left(X, v_{i}\right) \leq r, i=1, \ldots, n\right\}$.

For each nonnegative real $r$ the centdian problem can now be reformulated as follows,

$\min _{r}\{g(r)\}$

$g(r)=\lambda \cdot r+(1-\lambda) \cdot m(r)$.

Let $r^{*}$ be a minimum of the function $g(r)$. We will first identify explicitly a set $R$, called a finite dominating set, which includes $r^{*}$. To find $r^{*}$ we need to compute the objective function for all values of $r \in R$, and evaluate the minimum of the function over R (Mavrotas, 2009).

\subsection{Finding feasible solutions}

According to Tamir et al. (2003), we can identify a set of $R$ cardinality containing $r^{*}$, the optimal solution of the p-centdian problem. Therefore, we can find the initial candidate solution of restricted centdian problem by solving the $r$-restricted $p$-median problems for every $r \in R$. 
$R=R_{1} \cup R_{2} \cup R_{3}$,

where:

$$
\begin{aligned}
& R_{1}=\left\{u_{i} d\left(v_{i}, v_{j}\right) \mid v_{i}, v_{j} \in V\right\} \\
& R_{2}=\left\{d\left(v_{i}, v_{j}\right) /\left(1 / u_{i}+1 / u_{j}\right) \mid v_{i}, v_{j} \in V\right\} \\
& R_{3}=\left\{\left(d\left(v_{j}, v_{k}\right)-d\left(v_{i}, v_{k}\right)\right) /\left(1 / u_{j}-1 / u_{i}\right) \mid v_{i}, v_{j}, v_{k} \in V, v_{k} \in P\left(v_{i}, v_{j}\right)\right\}
\end{aligned}
$$

\subsection{Solution procedure for $r$-restricted p-median problem: first stage}

In this section, we present the algorithm to solve the $r$-restricted $p$-median problem. Let $P$ lbe the $r$ restricted $p$-median problem. The algorithm starts with an initial dominating set (ID) which is the set of $N$ nodes, the dominant set of the unconstrained median problem (Hakimi, 1964). The shortest distance matrix $D$ includes the shortest distances $d(i, j)$ for any node $i \in N$ and any candidate location $j \in I D$. The relaxed unconstrained median problem is solved using a Lagrangian relaxation for each iteration of the algorithm,

$$
\min _{X_{p} \subseteq D S} \sum_{i=1}^{n} w_{i} d\left(X_{p}, i\right) \text {, }
$$

where $X_{p}$ is the optimal solution of $\left(P^{\prime}\right)$ and it is also a feasible solution for $P 1$ we terminate the algorithm. Otherwise, the algorithm looks for the node that makes the most violation. Let node $v$ be the one, which has the longest distance from the closest facility in $X_{p}$, i.e.

$$
d\left(v, X_{p}\right)=\max _{i \in N} d\left(i, X_{p}\right) .
$$

Once node $v$ is identified, the set of ID is updated by adding all dominant points $D O M_{v}$ as follows,

$$
D O M_{v}=\{x \in G \mid d(x, v)=r\} \text {. }
$$

Now $D$ is updated by appending $\left|D O M_{v}\right|$ new columns, each corresponding to a point in $D O M_{v}$. We also add node $v$ to a set of $M V N$, which includes the most violated nodes. Note that $M V N=\phi$ in the beginning of the algorithm. In the modified distance matrix $D, d(i, j) \forall i \in N, j \in D S$ is defined as follows:

$d(i, j)= \begin{cases}M & \text { if } i \in V \text { and } d(i, j)>r \\ d(i, j) & \text { otherwise, }\end{cases}$

where $\mathrm{M}$ is a very large number. Formally the algorithm can be stated as follows:

\section{Algorithm 1}

Step 1: Set $I D=N, M V N=\phi, D$ is the shortest-distance matrix with elements $\{d(i, j), i \in N, j \in I D\}$.

Step 2: Solve problem $\left(P^{\prime}\right)$ to get the optimal solution, $X_{p}$.

Step 3: If all distance constraints are satisfied, STOP; $X_{p}$ is an optimal solution of $\left(P^{\prime}\right)$. Otherwise, find the most violated node $v$ according to Eq. (18). If $v \in V$, stop; no feasible solution exists for $\left(P^{\prime}\right)$.

Step 4: Set $I D=I D \cup D O M_{v}, M V N=M V N \cup v$. Append $\left|D O M_{v}\right|$ new columns to $D$ and compute or modify distances in $D$ according to Eq. (19). Return to Step 1.

\subsection{Lagrangian relaxation: second stage}

This section is intended to give an overview on how Lagrangian Relaxation is implemented to solve the unconstrained $p$-median problem. Consider the following mathematical problem, 


$$
\begin{array}{ll}
\min \sum_{j} \sum_{i} w_{j} d_{i j} y_{i j} \\
\text { subject to } & \\
\sum_{j \in J} y_{i j}=1 & , \forall i \\
y_{i j}-x_{i} \leq 0 & , \forall i, j \\
y_{i j}=\{0,1\} & , \forall i, j \\
x_{i}=\{0,1\} & , \forall i
\end{array}
$$

Lagrangian relaxation is based on the idea of removing all constraints and adding them to the objective function to make it easier to solve. Therefore, to solve a problem, Lagrangian relaxation removes a constraint but introduces a penalty for violating the removed constraint. This revised problem is then optimized accordingly. Next, we explain how the technique of Lagrangian relaxation is implemented to solve the bi-criteria problem:

\subsubsection{Setting up}

We remove constraint (21) and add the constraint and a vector of variables called Lagrange multipliers to the objective function. For this particular implementation, all Lagrange multipliers are initialized to an arbitrary number, e.g. 300, and compute the following,

$$
\max _{\lambda} \min _{x, y} \sum_{j} \sum_{j} w_{j} d_{i j} y_{i j}+\sum_{j} \lambda_{j}\left[1-\sum_{i} y_{i j}\right]
$$

\subsubsection{Solving the simplified problem}

For fixed values of the Lagrange multipliers, the objective function in the previous step is minimized by computing the value of setting each of the location variables, $X$, to 1 . Let we have,

$$
V_{i}=\sum_{j} \min \left\{0, w_{j} d_{i j}-\lambda_{j}\right\}
$$

for each candidate location $j$. The $P$ smallest values of $V$ is then determined and the corresponding location variables $(X)$ are set to 1 and all other location variables $(X)$ to 0 . The allocation variables $(Y)$ are then calculated by using the GreedyExp algorithm that will be described in the next section.

\subsubsection{Updating the lower bound and upper bound}

We consider an upper bound for the objective function, as an estimate of the worst-case scenario, and a lower bound, as an optimistic estimate for the best-case scenario, for each iteration of the algorithm. An upper bound is a solution which meets the constraints of the original unmodified problem. The minimum of the upper bound, the best guess for the worst case, is sought for the purposes of our proposed algorithm. The upper bound can be determined by simply determining the closest location to each customer. The corresponding allocation variables $(Y)$ are then set to 1 while all others are set to 0 . We then evaluate the P-Median objective function as stated originally.

Note that the solution to the simplified problem as outlined in Step 2 may or may not meet the constraints of the original problem. Since the modified problem need not meet the constraints of the original, the modified problem will produce a solution, which would always be better or equal to the solution of the original problem. Thus, a lower bound on the $P$-Median problem can be determined by simply evaluating the original $P$-Median objective function using the values for the variables determined in Step 2. 


\subsubsection{Modifying the Lagrange multipliers}

A technique, which drives the iterations to an optimal solution and meets the constraints of the original problem, is called sub-gradient optimization. We use this method to update the value of the Lagrange Multipliers. Based on the sub gradient optimization, a new variable $t$ is introduced as follows:

$$
t^{n}=\frac{A^{n}\left(U B-L^{n}\right)}{\sum_{j}\left\{\sum_{i} y_{i j}-1\right\}},
$$

where

$A^{n} \quad:$ a constant on the $n$th iteration, where $A^{1}$ is generally set to 4

$t^{n} \quad:$ the stepsize at the $n$th iteration of the Lagrangian procedure

UB : the best, the smallest and the upper bound on the $P$-median objective function

$L^{n} \quad$ : the value of the objective function using the solution obtained from the relaxed problem

$Y_{i j}^{n} \quad:$ the optimal value of the allocation variable, $Y_{i j}^{n}$ on the $n$th iteration

The Lagrange multipliers are then updated according the following equation

$$
\lambda_{j}^{n+1}=\max \left\{0, \lambda_{j}^{n}-t^{n}\left(\sum_{i} y_{i j}^{n}-1\right)\right\} .
$$

\subsubsection{Evaluating the results}

If, at any point in time, the lower bound is equal or very close to the upper bound, then the optimal solution to the original problem could now be available and the algorithm in this case terminates. To put a reasonable cap on the running time of the algorithm, we limit the number of iterations to 100 and the value of $A$ does not have to be less than 0.01 . If the upper bound does not decrease after 4 consecutive iterations, a new A is replaced by A / 2. If none of these stopping conditions is met, the implementation reiterates starting at Step 2.

\subsection{Computing optimal allocations: 3th stage}

We use GreedyExp algorithm proposed by Salcedo-Sanz et al. (2008) for assignment phase. This greedy approach starts from a permutation of demands $\pi^{*}$, order in which we assign the demands to facilities, which sorts the demands for their distance to the nearest facility. We start assigning demand nodes to facilities following the order given by permutation $\pi^{*}$. When a given demand node $i$ cannot be assigned to its nearest facility $k$ due to the capacity constraint, we calculate the distance of all the demand nodes in $k$ to the second nearest facility. If the distance of a demand node $j$ which is already assigned to $k$ to its second closest facility is smaller than the distance from demand node $i$ to its second closest facility, and $w_{j} \geq w_{i}$, then we reassign demand node $j$ to its second closest facility, and substitute it by demand node $i$. In the case that there is not such a demand node or with the requirements of distance or weight, demand node $i$ is assigned to its second closest facility.

\section{Computational results}

We divide this section to two parts. In the first part, we will validate the solution approach effectiveness by comparing the results of our algorithm for the single objective case with results obtained from GAMS. After validation of our proposed algorithm, we will employ it to solve for the bi-objective location-allocation model in the second part. The proposed model of this paper along with other comparative codes from the literature were coded using MATLAB R2008.a and they were run on a Pentium IV PC with $2 \mathrm{GHz}$ CPU and 256MB RAM. 


\subsection{Comparing heuristic method and GAMS}

Consider a tree network of demands with 6 nodes and 5 edges. Assume that there are 42, 35, 28, 50, 45 and 45 demand units in nodes of the network. We want to locate 2 new emergency facilities in this network where the first facility has 100 units capacity and the second one has 200 units capacity. The parameter of $\lambda$ is set to be 0.8 . The shortest distance matrix of the mentioned network is as follows,

$$
D=\left[\begin{array}{cccccc}
0 & 6 & 8 & 8 & 9 & 19 \\
6 & 0 & 2 & 2 & 0 & 13 \\
8 & 2 & 0 & 4 & 5 & 15 \\
8 & 2 & 4 & 0 & 1 & 11 \\
9 & 3 & 5 & 1 & 0 & 10 \\
19 & 13 & 15 & 11 & 10 & 0
\end{array}\right]
$$

Since we are only validating the proposed method in this section, we compare the results for the single objective centdian problem using GAMS and the results are summarized in Table 1.

\section{Table1}

The optimal results of the single objective centdian problem

\begin{tabular}{lcccccc}
\hline$r$ & $\begin{array}{l}\text { Centdian objective using the } \\
\text { proposed algorithm }\end{array}$ & $\begin{array}{l}\text { p-center objective of the } \\
\text { proposed algorithm }\end{array}$ & $\begin{array}{l}\text { p-median objective of } \\
\text { the proposed algorithm }\end{array}$ & $\begin{array}{l}\text { Centdian objective } \\
\text { using GAMS }\end{array}$ & $\begin{array}{l}\text { p-median objective } \\
\text { using GAMS }\end{array}$ & CPU(s) \\
\hline 7.5 & 21.6 & 7.5 & 78 & 21.6 & 78 & 0.228646 \\
8 & 21.8 & 8 & 77 & 21.8 & 78 & 0.218546 \\
9 & 22.4 & 9 & 76 & 22.4 & 78 & 0.158602 \\
9.5 & 22.6 & 9.5 & 75 & 22.6 & 75 & 0.604730 \\
10 & 22.6 & 10 & 73 & 22.6 & 73 & 0.570210 \\
11 & 23 & 11 & 71 & 23 & 71 & 0.150417 \\
13 & 24 & 13 & 68 & 24 & 68 & 0.151132 \\
15 & 25 & 15 & 65 & 25 & 65 & 0.151372 \\
19 & 27.8 & 19 & 63 & 27.8 & 63 & 0.136594 \\
\hline
\end{tabular}

The results show that the proposed heuristic mode of this paper performs relatively efficient.

\section{2. the bi-objective model}

In this section, for validation of our solution approach we have solved one of the best well-known problems in centdian problems literature using the proposed method this paper and we compared our results with results obtained by the exhaustive search algorithm presented by Perez-Brito et al. (1997). In this problem we want to locate 2 new facilities in a tree network with 6 demand nodes. The weights of all the nodes are equal to 1 and the network is shown in Fig. 1. Table 2 shows a comparison between our solution results and the final solution reported by Perez-Brito et al.'s method.

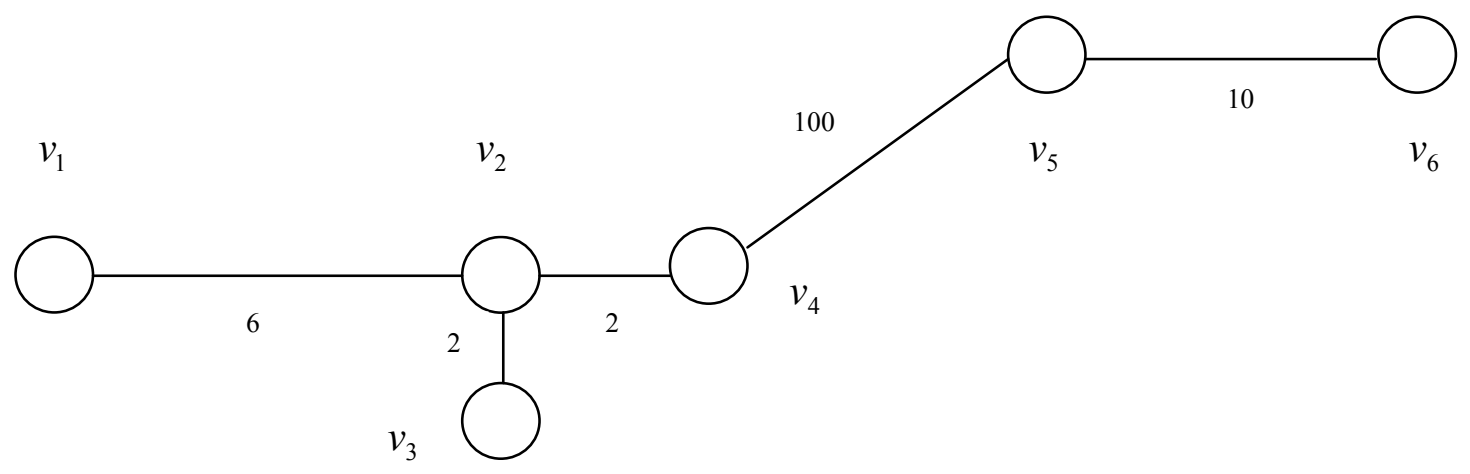

Fig. 1. The sample tree network 


\section{Table2}

The comparison between the results of the proposed method versus Perez-Brito et al.'s method

\begin{tabular}{lccccccc}
\hline \multirow{2}{*}{ Number of demand nodes } & \multirow{2}{*}{ Number of facilities } & \multicolumn{3}{c}{ Perez-Brito algorithm } & \multicolumn{3}{c}{ The proposed algorithm } \\
\cline { 2 - 8 } & & $f_{1}$ & $f_{2}$ & CPU(s) & $f_{1}$ & $f_{2}$ & CPU(s) \\
\hline 6 & 2 & 22 & 5 & 11.530907 & 22 & 5 & 7.435479 \\
10 & 3 & 58 & 12 & 68.801841 & 58 & 12 & 9.895077 \\
20 & 5 & 94 & 21.5 & 808.871928 & 94 & 21.5 & 143.165704 \\
30 & 8 & 202 & 23 & 1836.873544 & 198 & 25.5 & 675.558150 \\
\hline
\end{tabular}

As we can observe from the results, the proposed method of this paper needs significantly less amount of time compared with Perez-Brito algorithm. For small size instances, the proposed heuristic approach yields the exact optimal solution. For large instances, the proposed heuristic produces near optimal solutions in less amount of CPU time. For the implementation of the e-constraint, we must first find a range for the first objective function used as constraint. The most common approach is to calculate these ranges from the payoff table, the table with the results from the individual optimization of two objective functions. We will calculate the pay-off table for our sample problem.

\subsubsection{Calculating the pay-off table}

The payoff table for single objective model considering the sample network is shown in Table 3 .

Table3

Payoff table

\begin{tabular}{lll}
\hline & $\mathrm{f} 1$ & $\mathrm{f} 2$ \\
\hline Min f1 & 35.7 & 36.4 \\
Min f2 & - & 22 \\
\hline
\end{tabular}

\subsubsection{Result of e-constraint method}

After calculating the payoff table we will divide the ranges of the objective functions to four equal intervals and we use some grid points as the values of $e_{2}$ in the e-constraint method. The number of grid point is assumed to be equal with the number of $r$ values in centdian objective. Table 4 shows the details of the implementation of the proposed model on the bi-objective.

Table 4

Result of solving biobjective case for $\lambda=0.7$

\begin{tabular}{llll}
\hline interval & $\mathrm{f} 1$ & $\mathrm{f} 2$ & $\mathrm{CPU}(\mathrm{s})$ \\
\hline 1 & 35.7 & 22 & 0.148253 \\
2 & 35.6 & 24 & 0.425348 \\
5 & 35.2 & 32 & 0.460500 \\
\hline
\end{tabular}

We have calculated the Pareto optimal set for values $\lambda=0.5,0.3$ and 0.1 . The results are shown in Table 5.

\section{Table 5}

The results of the efficient solutions for different values of $\lambda$

\begin{tabular}{llllllllll}
\hline \multirow{2}{*}{ Interval } & \multicolumn{1}{c}{$\lambda=0.50$} & \multicolumn{3}{c}{$\lambda=0.30$} & \multicolumn{3}{c}{$\lambda=0.10$} \\
\cline { 2 - 9 } & $f_{1}$ & $f_{2}$ & $\mathrm{CPU}(\mathrm{s})$ & $f_{1}$ & $f_{2}$ & $\mathrm{CPU}(\mathrm{s})$ & $f_{1}$ & $f_{2}$ & CPU(s) \\
\hline 1 & 42.75 & 22 & 0.175520 & 56.85 & 22 & 0.439730 & 70.95 & 22 & 0.339511 \\
2 & 42.5 & 24 & 0.190233 & 56.3 & 24 & 0.346951 & 70.1 & 24 & 0.379007 \\
3 & 42.5 & 28 & 0.155257 & 55.9 & 28 & 0.257550 & 69.3 & 28 & 0.300535 \\
4 & 42.25 & 30 & 0.292608 & 55.35 & 30 & 0.503221 & 68.45 & 30 & 0.600141 \\
5 & 41.5 & 32 & 0.266935 & 54.1 & 32 & 0.466462 & 66.7 & 32 & 0.544720 \\
6 & 41 & 36 & 0.087322 & 53 & 36 & 0.150650 & 65 & 36 & 0.168793 \\
7 & 40.5 & 36 & 0.087808 & 51.5 & 36 & 0.174555 & 62.5 & 36 & 0.167462 \\
8 & 40 & 38 & 0.088321 & 50 & 38 & 0.132963 & 60 & 38 & 0.168239 \\
9 & 39.25 & 39 & 0.150417 & 49.8 & 39 & 0.259850 & 58.6 & 39 & 0.167027 \\
\hline
\end{tabular}


We can do a sensitivity analysis for different amounts of $\lambda$. Fig. 2 shows the results of two objective functions based on different values of $\lambda$.

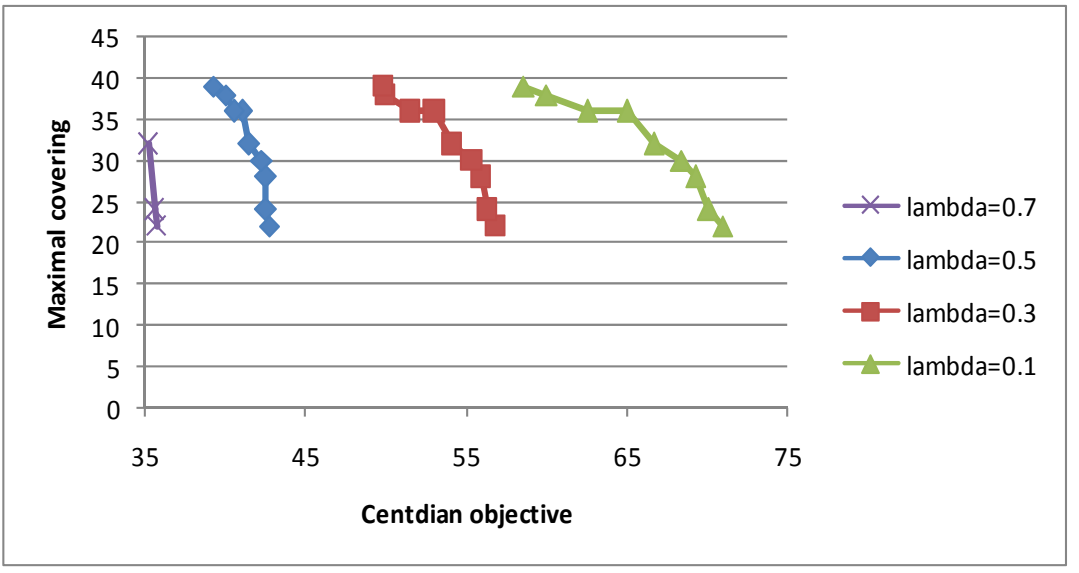

Fig. 2. Sensitivity analysis for $\lambda$

As we can see, the slope of the Pareto optimal curve will decrease as $\lambda$ decreases. This means that by decreasing $\lambda$, the weight of the center objective is decreased and the weight of the median objective increases. By increasing the weight of median function, the Pareto optimal points are farther apart and the slope decreased.

\section{Conclusions and future research}

In this paper, we have introduced a bi-objective model for emergency services location-allocation problem. The primary objective of the proposed model is that we assumed the new emergency facilities have capacity constraints. The advantages of the proposed model are as follows: considering maximum distance constraint and considering capacity constraints helps capture more realistic problems. To solve the proposed model we introduced new heuristic algorithm and then compared the solution obtained from our heuristic with solution obtained from GAMS. Our computational results showed that the algorithm is reasonably effective. As a future work, we could consider the demand uncertainty and reformulate the location-allocation problem. Also, it is interesting to develop some efficient meta-heuristic like NSGA II for generating the Pareto optimal set specially for large-scale problems.

\section{References}

Berman, O., Larson, R. C., \& Chiu, S. S. (1985). Optimal server location on a network operating as an M/G/1 queue. Operations Research, 33(4), 746-771.

Berman, O., \&Yang, K. E. (1991). Medi-Centre Location Problems. The Journal of the Operational Research Society, 42(4), 313-322.

Bianchi,C., \& Church, R. (1988). A hybrid FLEET model for emergency medical service system design. Social Sciences in Medicine, 26(1), 163-171.

Beraldi, P., \& Ruszczynski, A. (2002). A branch and bound method for stochastic integer problems under probabilistic constraints. Optimization Methods and Software, 17, 359-382.

Calvo, A. H., \& Marks, H. (1973). Location of health care facilities: an analytical approach. SocioEconomic Planning Sciences, 7, 407-422.

Carbone, R. (1974). Public facility location under stochastic demand. INFOR, 12, 261-270.

Colebrook, M., \& Sicilia, J. (2007). A polynomial algorithm for the multicriteria cent-dian location problem. European Journal of Operational Research, 179, 1008-1024.

Colebrook, M., \& Sicilia, J. (2006). An O(mn) algorithm for the anti-cent-dian problem. Applied Mathematics and Computation, 183, 350-364.

Daskin, M. S. (2008). What You Should Know About Location Modeling. Naval Research Logistics, $55,283-294$. 
Daskin, M. S. \& Stern, E. H. (1981). A hierarchical objective set covering model for emergency medical service vehicle deployment. Transportation Science, 15(2), 137-152.

Garfinkel, R. S., Neebe, A. W., \& Rao, M. R. (1977). The m-center problem: minimax facility location. Management Science, 23, 1133-1142.

Goldberg, J. and Paz, L. (1991) Locating emergency vehicle bases when service time depends on call location. Transportation Science, 25, 264-280.

Hakimi, S. L. (1964). Optimum Locations of Switching Centers and the Absolute Centers and Medians of a Graph. Operations Research, 12(3), 450-459.

Halpern, J. (1976). The location of a center-median convex combination on an undirected tree. Journal of regional science, 16(2), 237-245.

Halpern, J. (1978). Finding minimal center-median convex combination (Cent-Dian) of a Graph. Management Science, 24(5), 535-544.

Hooker, J. N., Garfinkel, R. S., \& Chen, C. K. (1991). Finite dominating sets for network location problems. Operations Research, 39(1), 100-118.

Hochbaum, D. S., \& Pathria, A. (1998). Locating centers in a dynamically changing network and related problems. Location Science, 6, 243-256.

Halpern, J. (1980). Duality in the cent-dian of a graph. Operations Research, 28(3), 722-735.

Jia, H., Ordonez, F., Dessouky, M. (2007). A modeling framework for facility location of medical services for large-scale emergencies. IIE Transactions, 39, 41-55.

Paluzzi, M. (2004). Testing a heuristic P-median location allocation model for siting emergency service facilities. presented at the Annual Meeting of the Association of American Geographers, Philadelphia, PA.

Perez-Brito, D., Moreno-Perez, J. A., \& Rodriguez-Martin, I. (1997). Finite Dominating Set for the pFacility Cent-Dian Network Location Problem. Studies in Location Analysis, 11, 27-40.

Perez-Brito, D., Moreno-Perez, J. A., \& Rodriguez-Martin, I. (1998). The 2-facility centdian network problem. Location Science, 1998. 6, 369-381.

Mirchandani, P. B. (1987). Generalized hierarchical facility location. Transportation Science, 21, 123-125.

Mirchandani, P. B. (1980). Locational decisions on stochastic networks. Geographical Analysis, 12 , 172-183.

ReVelle, C., \& Hogan, K. (1989). The maximum availability location problem. Transportation Science, 23, 192-200.

Salcedo-Sanz, S., Portilla-Figueras, J. A., Ortiz-Garciia, E. G., Peirez-Bellido, A. M., Thraves, C., Fernandez-Anta, A., \& Yao, X. (2008). Optimal switch location in mobile communication networks using hybrid genetic algorithms. Applied Soft Computing, 8, 1486-1497.

Schilling, D.A. (1982). Strategic facility planning. the analysis of options. Decision Sciences, 13, 1-14.

Serra, D., \& Marianov, V. (1999). The P-median problem in a changing network: the case of Barcelona, Location Science, 6(1), 383-394.

Storbeck, J. (1989). Slack, natural slack, and location coveting, Socio-Economic Planning Sciences 16(3).

Talwar, M. (2002). Location of rescue helicopters in South Tyrol, presented at the 37th Annual ORSNZ Conference, Auckland, New Zealand.

Tamir, A., Brito, D. P., \& Moreno-Perez, J. A. (2003). A Polynomial Algorithm for the p-Centdian Problem on a Tree.

Tien, J. M., El-Tell, K., \& Simons, G. (1983). Improved formulations to the hierarchical health facility location-allocation problem, IEEE Transactions in Systems Management and Cybernetics, $13,1128-1132$.

Toregas, C., Swain, R., ReVelle, C., \& Bergman, L. (1971). The location of emergency service facility. Operations Research, 19, 1363-1373. 